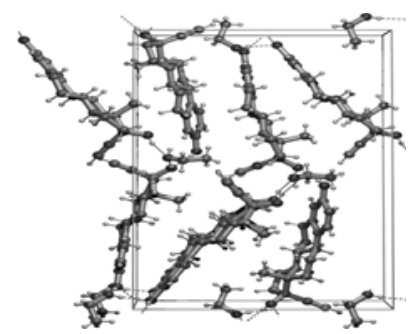

Figure 1. Crystal structure of ethinyl estradiol ethanolate

For ethinyl estradiol we determined crystal structures of several unknown solvates by single-crystal X-ray diffraction.

Ethinyl estradiol shows a remarkable flexibility in forming distinctly different hydrogen bonding patterns, resulting in a diverse set of solvate structures. The structural aspects and scope of solvate formation of ethinyl estradion is discussed and compared to other related estrogens.

[1] Gu C.H.; Li H.; Gandhi R.B.; Raghavan K., Int.J.Pharm., 2004, 283, 117.

[2] Nangia, A.; Desiraju, G.R., Chemm. Commun. 1999, 605

\section{MS20 P16}

Crystal structure of penicillin binding protein 4 (dacB) from Escherichia coli. S-Y. Park, J.R.H. Tame, Protein Design Laboratory, Yokohama City University, Suehiro 1-7-29, Tsurumi-ku, Yokohama 230-0045, Japan

E-mail: park@tsurumi.yokohama-cu.ac.jp
Keywords: antibiotics, crystallography, protein crystals

The bacterial cell wall is a single molecule of peptidoglycan, which is essential for cell growth and survival under normal conditions. Since the enzymes involved in peptidoglycan synthesis have no counterpart in mammalian biochemistry, they present a variety of attractive targets for antibiotic design. Many natural bacteriocidal compounds, including the penicillin family, also exploit the dependence of bacterial survival on the integrity of the cell wall.

The crystal structure is presented of penicillin binding protein 4 (PBP4) from Escherichia coli, a bifunctional enzyme with both DD-endopeptidase and DDcarboxypeptidase activity. PBP4 is one of 12 penicillin binding proteins in E.coli involved in the synthesis and maintenance of the cell wall. The model contains a penicillin binding domain similar to known structures, but includes a large insertion which folds into domains with unique folds. The structures of the protein covalently attached to five different antibiotics presented here show the active site residues are unmoved compared to the apo protein, but nearby surface loops and helices are displaced in some cases. Movement of conserved residues suggests a possible cause for the slow deacylation rate of PBP4[1].

[1] H. Kishida, S. Unzai, D. I Roper, A. Lloyd, S.-Y. Park, J.R.H. Tame. Biochemistry 2006, 45, 783 